\title{
Modelagem da dispersão de poluentes na atmosfera combinando o ADMM com homogeneização matemática
}

Modeling pollutant dispersion in the atmosphere by combining the ADMM with mathematical homogenization

\author{
K. Rui ${ }^{1 *}$; C. P. Costa² L. D. Pérez-Fernández ${ }^{2}$; J. Bravo-Castillero ${ }^{3}$ \\ ${ }^{1}$ Programa de Pós-Graduação em Engenharia Mecânica, Universidade Federal do Rio Grande do Sul, CEP 90050- \\ 170, Porto Alegre-RS, Brasil \\ ${ }^{2}$ Instituto de Física e Matemática/Departamento de Matemática e Estatística, Universidade Federal de Pelotas, CEP \\ 96010-971, Pelotas-RS, Brasil \\ ${ }^{3}$ Facultad de Matemática y Computación, Universidad de La Habana, CP 10400, La Habana, Cuba
}

*karinerui@gmail.com

(Recebido em 11 de abril de 2017; aceito em 22 de maio de 2017)

\begin{abstract}
O método multicamadas de advecção-difusão (ADMM) produz soluções semianalíticas precisas dos problemas de valores de contorno/iniciais para equações de advecção-difusão com coeficientes variáveis que modelam a dispersão de poluentes na atmosfera, e apresenta o menor custo computacional quando comparado com outros métodos baseados em transformadas integrais. Contudo, em situações operativas tais como desastres naturais/industriais que resultam na fuga de poluentes na atmosfera, é necessário aferir rapidamente e com exatidão a distribuição da concentração dos poluentes no nível do solo para minimizar o impacto na saúde e na economia. Aqui, para acelerar a disponibilidade de resultados com mínima perda de precisão, o ADMM é combinado com homogeneização matemática, cujo emprego na modelagem de dispersão de poluentes parece ser novidade. A abordagem proposta é comparada com a aplicação direta do ADMM e às observações do experimento de Hanford para avaliar a exatidão das estimativas, assim como sua eficiência computacional, considerando condições atmosféricas estáveis. Os resultados mostram que a combinação do ADMM com a homogeneização matemática apresenta uma redução significativa no custo de tempo computacional.
\end{abstract}

Palavras-chave: modelagem da dispersão de poluentes na atmosfera, método multicamadas de advecção-difusão, homogeneização matemática

The multilayer advection-diffusion method (ADMM) produces accurate semi-analytical solutions of boundary/initial value problems for advection-diffusion equations with variable coefficients that model pollutant dispersion in the atmosphere, and presents the lowest computational cost when compared to others methods based on integral transformations. However, in operational situations such as natural/industrial disasters resulting in the escape of pollutants into the atmosphere, it is necessary to quickly and accurately measure the concentration distribution of pollutants at ground level to minimize the impact on health and the economy. Here, to accelerate the availability of results with minimal loss of precision, the ADMM is combined with mathematical homogenization, whose use in the modeling of pollutant dispersion seems to be new. The proposed approach is compared with the direct application of ADMM and the observations of the Hanford experiment to evaluate the accuracy of the estimates as well as their computational efficiency considering stable atmospheric conditions. The results show that the combination of ADMM with mathematical homogenization presents a significant reduction in computational time cost.

Keywords: modeling the dispersion of pollutants in the atmosphere, advection-diffusion multilayer method, mathematical homogenization

\section{INTRODUÇÃO}

O método multicamadas de advecção-difusão (ADMM, siglas em inglês - ver [1]) é um dos métodos baseados em transformadas integrais utilizados para resolver analiticamente os problemas de valores de contorno/iniciais para equações de advecção-difusão com coeficientes variáveis que modelam a dispersão de poluentes na atmosfera. Foi relatado em [2] que o 
ADMM produz estimativas da concentração de poluentes tão exatas quanto as produzidas mediante outros métodos baseados em transformadas integrais tais como o GILTT (siglas em inglês para técnica da transformada integral de Laplace generalizada - ver [3]), mas com custo computacional significativamente menor. Esta última característica é essencial em situações operativas tais como em desastres naturais/industriais nas quais estimativas precisas e rápidas (idealmente em tempo real) da distribuição e concentração a nível do solo dos poluentes liberados na atmosfera. Assim, a disponibilidade de estimativas rápidas e precisas em condições operativas é necessária para a prevenção e diminuição do impacto de emergência de poluição tanto na saúde quanto na economia.

O ADMM considera a aproximação constante por partes dos coeficientes contínuos do perfil de velocidade do vento e da difusividade turbulenta na direção vertical $z \in[0, h]$ ( $h$ é a altura da camada limite planetária (CLP)), e a aplicação da transformada de Laplace. Tal aproximação é a média local dos coeficientes variáveis em cada subcamada. Dessa forma, o problema original com coeficientes contínuos é aproximado por um problema com coeficientes constantes por partes que produzirá resultados mais precisos e com maior custo computacional a medida que a discretização da CLP for mais fina.

Neste trabalho, para acelerar a disponibilidade de resultados com a menor perda de exatidão possível, o ADMM é combinado com homogeneização matemática [4,5,6]. De forma resumida, a homogeneização de meios heterogêneos baseia-se nas hipóteses de separação de escalas estruturais e do contínuo. Isto é, a estrutura do meio (aqui, a CLP) é caracterizada por um parâmetro geométrico pequeno $\varepsilon$ definido como o quociente dos comprimentos característicos de ambas as escalas microscópica e macroscópica, de maneira que propriedades efetivas ou macroscópicas podem lhe ser atribuídas ao meio heterogêneo (sempre que as condições de contorno sejam uniformes). Com tais considerações, a hipótese de homogeneidade equivalente é válida, ou seja, existe um meio homogêneo ideal que é equivalente ao meio heterogêneo no sentido de que as propriedades físicas do primeiro são as propriedades efetivas do segundo. Do ponto de vista matemático, a hipótese de homogeneidade equivalente é válida se a solução do problema original (aqui, do ADMM) com coeficientes variáveis (aqui, constantes por partes), que modela o meio heterogêneo (aqui, a CLP), converge para a solução do chamado problema homogeneizado com coeficientes constantes, que modela o meio homogêneo associado, na norma do espaço em que tais soluções são procuradas quando $\varepsilon \rightarrow 0^{+}$. Em outras palavras, a norma da diferença de tais soluções é de ordem de uma potência de $\varepsilon$. Aqui, os coeficientes efetivos da difusividade turbulenta vertical e do perfil da velocidade média do vento são obtidos a partir do problema do ADMM no espaço de Laplace para condições estáveis. Segundo nosso melhor conhecimento, a aplicação da homogeneização matemática à modelagem da dispersão de poluentes na atmosfera parece ser uma novidade na área.

Este trabalho está organizado da seguinte forma: a seção 2 contém a formulação do problema original; as seções 3 e 4 descrevem os processos de solução do problema via o ADMM na sua forma pura e em combinação com a homogeneização matemática, respectivamente; resultados de várias simulações são apresentados e discutidos na seção 5; e as conclusões finais são declaradas na seção 6 .

\section{FORMULAÇÃO DO PROBLEMA}

Considere o sistema de coordenadas cartesiano Oxyz tal que a fonte de poluentes está localizada no ponto $\left(0,0, H_{s}\right)$ e a direção do vento é paralela ao eixo $x$. Nesta abordagem inicial da homogeneização matemática na modelagem da dispersão de poluentes, considera-se o modelo estacionário bidimensional obtido da equação de advecção-difusão de [7] ao integrar lateralmente e desprezar a difusão longitudinal.

O modelo descrito acima é formulado como segue: dados $h, Q \in \mathbb{R}_{+}^{*}, H_{s} \in(0, h)$, e $u, K \in C([0, h])$, encontrar $\overline{c^{y}} \in \mathcal{H}^{1}\left(\mathbb{R}_{+} \times[0, h]\right)$ tal que 


$$
\begin{aligned}
& u(z) \frac{\partial \overline{c^{y}}}{\partial x}=\frac{\partial}{\partial z}\left[K(z) \frac{\partial \overline{c^{y}}}{\partial z}\right], x \in \mathbb{R}_{+}^{*}, z \in(0, h), \\
& \left.K(0) \frac{\partial \overline{c^{y}}}{\partial z}\right|_{z=0}=\left.K(h) \frac{\partial c^{y}}{\partial z}\right|_{z=h}=0, x \in \mathbb{R}_{+}^{*}, \\
& u(z) \overline{c^{y}}(0, z)=Q \delta\left(z-H_{s}\right), z \in(0, h),
\end{aligned}
$$

em que $u(z)$ é a velocidade média do vento, $K(z)$ é a difusão turbulenta, $\overline{c^{y}}(x, z)$ é a concentração média de poluentes integrada lateralmente, $C(\cdot)$ é o espaço das funções contínuas, $\mathcal{H}^{1}(\cdot)$ é o espaço das funções de quadrado integrável cujas derivadas de primeira ordem são de quadrado integrável, $Q$ é a taxa de emissão da fonte poluidora, e $\delta(\cdot)$ é a função delta de Dirac. Para simplificar a notação, escreve-se $c(x, z)=\overline{c^{y}}(x, z)$. Neste trabalho, consideram-se as seguintes parametrizações de $K(z)$ e $u(z)$ de [8] e [9], respectivamente:

$$
\frac{K(z)}{u_{*} h}=0.13\left(\frac{z}{h}\right)^{4 / 5}\left(1-\frac{z}{h}\right)
$$

em que $u_{*}$ é a velocidade de atrito, e

$$
\frac{u(z)}{u_{1}}=\left(\frac{z}{z_{1}}\right)^{p}
$$

em que $u_{1}$ é a velocidade do vento na altura $z=z_{1}$, e $p$ está relacionado à intensidade da turbulência [10].

Observe que as condições de contorno (2) serão satisfeitas se $K(0)=K(h)=0$, que é de fato o caso da parametrização (4) e de muitas outras na literatura. Contudo, como notado em [11], $K(z)$ deve ser não nulo para que a difusão vertical ocorra, ou seja, $K(0) \neq 0$. Observe também que a parametrização (5) é tal que $u(0)=0$, de maneira que condição de contorno (3) poderá ser satisfeita admitindo concentração de poluentes não nula na base da fonte poluidora, ou seja, $c(0,0) \neq 0$, o que contradiz a física do problema. Ainda, a condição contorno (3) rescrita como $c(0, z)=Q \delta\left(z-H_{s}\right) / u(z)$ produz uma forma indeterminada do tipo $0 / 0 \mathrm{em}$ $z=0$, que de fato converge para $c(0,0)=0$ quando $z \rightarrow 0^{+}$, pois $\delta\left(-H_{s}\right)=0$ e $u(z) \neq 0$ na vizinhança de $z=0$. Por outro lado, tais irregularidades são corrigidas de maneira natural pelo $\mathrm{ADMM}$ ao tomar aproximações constantes por partes de $K(z)$ e $u(z)$ definidas pelos seus valores médios locais como se verá a seguir.

\section{APLICAÇÃO DO ADMM}

Seja $\left\{z_{n}\right\}_{n=\overline{0, N}} \subset[0, h], \quad N \in \mathbb{N}, z_{n}=n \Delta z$, uma partição uniforme da CLP. Em cada subcamada $\left(z_{n-1}, z_{n}\right), n=\overline{1, N}$, de espessura $\Delta z=z_{n}-z_{n-1}$ da CLP, considere os valores médios de $K(z)$ e $u(z)$ :

$$
K_{n}=\frac{1}{\Delta z} \int_{z_{n-1}}^{z_{n}} K(z) d z \text { e } u_{n}=\frac{1}{\Delta z} \int_{z_{n-1}}^{z_{n}} u(z) d z
$$

que definem aproximações constantes por partes de $K(z)$ e $u(z)$. 
Seja $c_{n}(x, z)=c(x, z),(x, z) \in \mathbb{R}_{+}^{*} \times\left(z_{n-1}, z_{n}\right), n=\overline{1, N}$. Assim, o problema original (1)-(3) é aproximado pelo problema

$$
\begin{aligned}
& u_{n} \frac{\partial c_{n}}{\partial x}=K_{n} \frac{\partial^{2} c_{n}}{\partial z^{2}}, x \in \mathbb{R}_{+}^{*}, z \in\left(z_{n-1}, z_{n}\right), n=\overline{1, N}, \\
& c_{n}\left(x, z_{n}\right)=c_{n+1}\left(x, z_{n}\right), x \in \mathbb{R}_{+}^{*}, n=\overline{1, N-1}, \\
& \left.K_{n} \frac{\partial c_{n}}{\partial z}\right|_{z=z_{n}}=\left.K_{n+1} \frac{\partial c_{n+1}}{\partial z}\right|_{z=z_{n}}, x \in \mathbb{R}_{+}^{*}, n=\overline{1, N-1}, \\
& \left.\frac{\partial c_{1}}{\partial z}\right|_{z=0}=\left.\frac{\partial c_{N}}{\partial z}\right|_{z=h}=0, x \in \mathbb{R}_{+}^{*}, \\
& c_{n}(0, z)=\frac{Q}{u_{n}} \delta\left(z-H_{s}\right) \delta_{n \bar{n}}, z \in\left(z_{n-1}, z_{n}\right), n=\overline{1, N},
\end{aligned}
$$

em que condições (8) e (9) impõem a continuidade nos pontos da partição $z=z_{n}, n=\overline{1, N-1}$, à concentração de poluentes e ao fluxo turbulento, respectivamente. Ainda, $\delta_{n \bar{n}}$ na condição de contorno (11) é a delta de Kronecker ( $\delta_{n \bar{n}}=1$ se $n=\bar{n}, \delta_{n \bar{n}}=0$ se $n \neq \bar{n}$ ), em que $n=\bar{n}$ indica a subcamada da CLP em que a fonte poluidora está localizada, ou seja, $H_{s} \in\left(z_{\bar{n}-1}, z_{\bar{n}}\right)$.

De aplicar a transformada de Laplace $\mathcal{L}[\cdot]$ em relação à variável $x$ ao problema ADMM dado por (7)-(11), segue que, para cada $s \in \mathbb{C}$,

$$
\begin{aligned}
& \frac{d^{2} \varsigma_{n}}{d z^{2}}-\frac{u_{n} s}{K_{n}} \varsigma_{n}(s, z)=-\frac{Q}{K_{n}} \delta\left(z-H_{s}\right) \delta_{n \bar{n}}, z \in\left(z_{n-1}, z_{n}\right), n=\overline{1, N}, \\
& \varsigma_{n}\left(s, z_{n}\right)=\varsigma_{n+1}\left(s, z_{n}\right), n=\overline{1, N-1}, \\
& \left.K_{n} \frac{d \varsigma_{n}}{d z}\right|_{z=z_{n}}=\left.K_{n+1} \frac{d \varsigma_{n+1}}{d z}\right|_{z=z_{n}}, n=\overline{1, N-1}, \\
& \left.\frac{d \varsigma_{1}}{d z}\right|_{z=0}=\left.\frac{d \varsigma_{N}}{d z}\right|_{z=h}=0,
\end{aligned}
$$

em que $\varsigma_{n}(s, z)=\mathcal{L}\left[c_{n}(x, z)\right]$. Logo, para $z \in\left(z_{n-1}, z_{n}\right), n=\overline{1, N}$, a solução do problema ADMM no espaço de Laplace dado por (12)-(15) é

$$
\varsigma_{n}(s, z)=A_{n} \exp \left\{R_{n}(s) z\right\}+B_{n} \exp \left\{-R_{n}(s) z\right\}-\frac{Q \operatorname{senh}\left\{R_{n}(s)\left(z-H_{s}\right)\right\}}{K_{n} R_{n}(s)} H\left(z-H_{s}\right) \delta_{n \bar{n}},
$$

em que $R_{n}(s)=\sqrt{u_{n} s / K_{n}}$ e $H(\cdot)$ é a função de Heaviside. Os coeficientes constantes $A_{n}$ e $B_{n}$ em (16) são obtidos resolvendo o sistema de equações algébricas lineares que resulta de substituir (16) nas condições (13)-(15).

Logo, a solução do problema ADMM (7)-(11) segue de aplicar a transformada de Laplace inversa $\mathcal{L}^{-1}[\cdot]$ a (16). Note que a complexidade da solução (16) requer inverter numericamente a transformada de Laplace, de maneira que a solução final é semianalítica. O algoritmo de inversão mais utilizado é a quadratura de Gauss, mas aqui utiliza-se o algoritmo Fixed-Talbot [12] que é computacionalmente mais robusto (também, ver [13]). Portanto, para $z \in\left(z_{n-1}, z_{n}\right)$, $n=\overline{1, N}$, a solução $c_{n}(x, z)=\mathcal{L}^{-1}\left[\varsigma_{n}(s, z)\right]$ do problema ADMM (7)-(11) é

$$
c_{n}(x, z)=\frac{r}{M}\left[\frac{1}{2} \varsigma_{n}(r, z) \exp \{r x\}+\sum_{k=1}^{M-1} \operatorname{Re}\left\{\exp \left\{x S\left(\theta_{k}\right)\right\} \varsigma_{n}\left(S\left(\theta_{k}\right), z\right)\left(1+i \omega\left(\theta_{k}\right)\right)\right\}\right] \text {, }
$$


em que $r \in \mathbb{R}_{+}^{*}$ é fixo, $i=\sqrt{-1}$, e $S\left(\theta_{k}\right)=r \theta_{k}\left(\cot \theta_{k}+i\right), \omega\left(\theta_{k}\right)=\theta_{k}+\left(\theta_{k} \cot \theta_{k}-1\right) \cot \theta_{k}$, e $\theta_{k}=k \pi / M \in(-\pi, \pi)$ (para mais detalhes, ver [12]).

\section{HOMOGENEIZAÇÃO MATEMÁTICA} é

O problema homogeneizado associado ao problema ADMM no espaço de Laplace (12)-(15)

$$
\begin{aligned}
& \frac{d^{2} \bar{\zeta}}{d z^{2}}-\frac{\hat{u} s}{\hat{K}} \bar{\zeta}(s, z)=-\frac{Q}{\hat{K}} \delta\left(z-H_{s}\right), z \in(0, h), \\
& \left.\frac{d \bar{\zeta}}{d z}\right|_{z=0}=\left.\frac{d \bar{\zeta}}{d z}\right|_{z=h}=0
\end{aligned}
$$

em que $\bar{\zeta}(s, z)$ é tanto o valor médio de $\varsigma(s, z)$ quanto a transformada de Laplace da aproximação $\bar{c}(x, z)$ obtida via homogeneização matemática da solução do problema ADMM (7)-(11), $\bar{\zeta}(s, z)=\mathcal{L}[\bar{c}(x, z)]$, e os coeficientes efetivos $\hat{u}$ e $\hat{K}$ são aproximados pelas médias aritmética e harmônica, respectivamente, das aproximações constantes por partes de $u(z)$ e $K(z)$, ou seja,

$$
\hat{K}^{-1}=\frac{1}{h} \int_{0}^{h} \frac{d z}{K(z)} \approx \frac{1}{h} \int_{0}^{h} \frac{d z}{\underline{K}(z)}=\frac{\Delta z}{h} \sum_{n=1}^{N} K_{n}^{-1},
$$

e

$$
\hat{u}=\frac{1}{h} \int_{0}^{h} u(z) d z \approx \frac{1}{h} \int_{0}^{h} \underline{u}(z) d z=\frac{\Delta z}{h} \sum_{n=1}^{N} u_{n} .
$$

Para justificar matematicamente a formulação do problema homogeneizado (18)-(21), observe que, para cada $s \in \mathbb{C}$ e cada $N \in \mathbb{N}$, o problema ADMM no espaço de Laplace (12)(15) pode ser escrito como

$$
\begin{aligned}
& -\frac{d}{d z}\left[\underline{K}(z) \frac{d \varsigma}{d z}\right]+\underline{u}(z) s \varsigma(s, z)=Q \delta\left(z-H_{s}\right), z \in(0, h) \backslash\left\{z_{n}\right\}, n=\overline{1, N-1}, \\
& \llbracket \varsigma(s, z) \|_{z=z_{n}}=0, n=\overline{1, N-1}, \\
& \llbracket \underline{K}(z) \frac{d \varsigma}{d z} \|_{z=z_{n}}=0, n=\overline{1, N-1}, \\
& \left.\frac{d \varsigma}{d z}\right|_{z=0}=\left.\frac{d \varsigma}{d z}\right|_{z=h}=0
\end{aligned}
$$

em que ${ }_{z=z_{n}}$ denota o operador de salto nos pontos de descontinuidade $z=z_{n}$ de $\underline{K}(z)=K_{n}$ e $\underline{u}(z)=u_{n}, z \in\left(z_{n-1}, z_{n}\right)$, e as derivadas são consideradas no sentido generalizado (ver, por exemplo, o Capítulo 1 de [6]. Este problema tem uma solução generalizada $\varsigma(s, z)=\underline{\varsigma}(s, z)$ cuja existência pode ser provada mediante um princípio do máximo (por exemplo, o Lema 1.1, página 95, de [14]). Tomando o parâmetro geométrico que descreve a separação de escalas como $\varepsilon=1 / N$, tem-se que $N \rightarrow \infty$ é equivalente a $\varepsilon \rightarrow 0^{+}$, isto é, o limite no qual se baseia a homogeneização matemática. De aplicar o Lema 4.1, página 63, de [15], segue que se existem $\hat{K}, \hat{u} \in \mathbb{R}_{+}^{*}$ e $\bar{\varsigma} \in \mathcal{H}^{1}(\mathbb{C} \times[0, h])$ tais que $\underline{K}^{-1}(z) \rightarrow \hat{K}^{-1}, \underline{u}(z) \rightarrow \hat{u}$, e $\underline{\varsigma}(s, z) \rightarrow \bar{\zeta}(s, z)$ quando $N \rightarrow \infty$, então, para cada $s \in \mathbb{C}, \bar{\zeta}(s, z)$ é solução de (18) e $\underline{K}(z) d \underline{\varsigma} / d z \rightarrow \hat{K} d \bar{\zeta} / d z$ quando $N \rightarrow \infty$, o qual é aplicado para obter as condições de contorno (19). Por outro lado, para o caso puramente difusivo, em [16] provou-se, mediante uma abordagem que envolve 
funções de Green e teoremas de valor médio, uma fórmula cuja versão unidimensional é $\hat{K}=\left\langle\underline{K}^{-1}(z)\right\rangle^{-1}$, em que $\langle\cdot\rangle=h^{-1} \int_{0}^{h}(\cdot) d z$ é o operador de valor médio, que coincide com a fórmula (20) para o coeficiente efetivo da difusividade turbulenta. Ainda, se o domínio é $\varepsilon$ periódico, o Teorema 2.6 , página 33 , de [17] garante que $\underline{K}^{-1}(y) \rightarrow\left\langle\underline{K}^{-1}(y)\right\rangle_{Y}$ e $\underline{u}(y) \rightarrow\langle\underline{u}(y)\rangle_{Y}$ quando $N \rightarrow \infty$, com o operador de valor médio $\langle\cdot\rangle_{Y}=|Y|^{-1} \int_{Y}(\cdot) d y$ definido localmente sobre o período estrutural $Y$ э $y=z / \varepsilon$. Dado que, neste caso, o valor médio local coincide com o valor médio sobre todo o domínio, é possível identificar $\langle\cdot\rangle \operatorname{com}\langle\cdot\rangle_{Y}$, o qual leva a tomar $\hat{K}=\left\langle\underline{K}^{-1}(z)\right\rangle^{-1}$ e $\hat{u}=\langle\underline{u}(z)\rangle$, que no caso unidimensional se transformam nas fórmulas (20) e (21) para os coeficientes efetivos.

Logo, sendo $\hat{R}(s)=\sqrt{\hat{u} s / \hat{K}}$, a solução do problema homogeneizado no espaço de Laplace definido por (18)-(21) é

$$
\bar{\zeta}(s, z)=\frac{Q}{\hat{K} \hat{R}(s)}\left\{\frac{\cosh \{\hat{R}(s) z\}}{\operatorname{senh}\{\hat{R}(s) h\}} \cosh \left\{\hat{R}(s)\left(h-H_{s}\right)\right\}-\operatorname{senh}\left\{\hat{R}(s)\left(h-H_{s}\right)\right\} H\left(z-H_{s}\right)\right\} .
$$

Dessa forma, a aproximação por homogeneização matemática da solução do problema ADMM (7)-(11) segue de aplicar a transformada de Laplace inversa a (26). Como na seção anterior, devido à complexidade da solução (26), é necessário inverter numericamente a transformada de Laplace, de maneira que a aproximação final da solução é semianalítica, para o qual utilizamos o algoritmo Fixed-Talbot de [12]. Assim, a aproximação por homogeneização matemática da solução do problema ADMM (7)-(11) é

$$
\bar{c}(x, z)=\frac{r}{M}\left[\frac{1}{2} \bar{\zeta}(r, z) \exp \{r x\}+\sum_{k=1}^{M-1} \operatorname{Re}\left\{\exp \left\{x S\left(\theta_{k}\right)\right\} \bar{\zeta}\left(S\left(\theta_{k}\right), z\right)\left(1+i \omega\left(\theta_{k}\right)\right)\right\}\right] .
$$

\section{RESULTADOS E DISCUSSÃO}

Para avaliar o desempenho da abordagem proposta combinando o ADMM e homogeneização matemática dada por (26)-(27), foram realizadas simulações computacionais cujos resultados foram comparados com os resultados da aplicação direta do ADMM dada por (16)-(17), e com as observações do experimento de Hanford [18]. Os parâmetros usados no algoritmo de inversão numérica da transformada de Laplace são $M=100$ e $r=2 M / 901 x$.

Este experimento foi realizado em Hanford, uma região semiárida no sudeste de Washington (EUA), no período de 18/05/1983 a 27/06/1983. A superfície do terreno é plana com vegetação de gramíneas de $1 \mathrm{a} 2 \mathrm{~m}$ de altura e comprimento de rugosidade de $z_{0}=0.03 \mathrm{~m}$. O experimento consistiu em liberar o traçador hexafluoreto de enxofre $\left(\mathrm{SF}_{6}\right)$, de uma torre com vazão de $Q=0.3 \mathrm{~g} / \mathrm{s}$ a uma altura de $H_{s}=2 \mathrm{~m}$. Foram conduzidos seis experimentos. Os dados foram coletados a $1.5 \mathrm{~m}$ do solo em cinco arcos de amostragem localizados a distâncias de $100 \mathrm{~m}$, $200 \mathrm{~m}, 800 \mathrm{~m}, 1600 \mathrm{~m}$ e $3200 \mathrm{~m}$ a partir da fonte com distância angular de $8^{\circ}, 4^{\circ}, 4^{\circ}, 2^{\circ}$ e $3^{\circ}$, respectivamente. Uma torre meteorológica de $122 \mathrm{~m}$ foi instalada a $100 \mathrm{~m}$ ao norte da área de liberação com sensores de velocidade e direção do vento, e temperatura em altitudes de $2.1 \mathrm{~m}$, $9.1 \mathrm{~m} ; 5.2 \mathrm{~m}, 30.5 \mathrm{~m}, 61 \mathrm{~m}, 91 \mathrm{~m}$ e $122 \mathrm{~m}$ para monitorar as condições durante as liberações dos traçador para garantir que o material fosse lançado durante períodos de condições de vento e de estabilidade adequadas. As liberações ocorreram durante condições de moderadamente estáveis a quase-neutras.

Figura 1 apresenta os perfis horizontais da concentração de poluentes próxima do nível do solo resultados das simulações computacionais de ambos os métodos em comparação com as observações do experimento de Hanford para $p=0.35$ e $z_{1}=10 m$ em (5). Nas diversas 
simulações realizadas observou-se que a aplicação direta do ADMM (16)-(17) consumiu de 15 a 20 vezes mais tempo computacional que a combinação do ADMM com a homogeneização matemática (26)-(27). Este fato é relevante quando se lida com uma grande quantidade de dados. Por outro lado, observa-se na Fig. 1 que ambas as curvas têm o mesmo comportamento qualitativo, sendo que a abordagem proposta subestima (resp. superestima) os valores da concentração de poluentes em regiões próximas (resp. afastadas) da fonte poluidora. Mesmo assim, a abordagem proposta fornece boas aproximações da concentração em comparação com as observações experimentais.

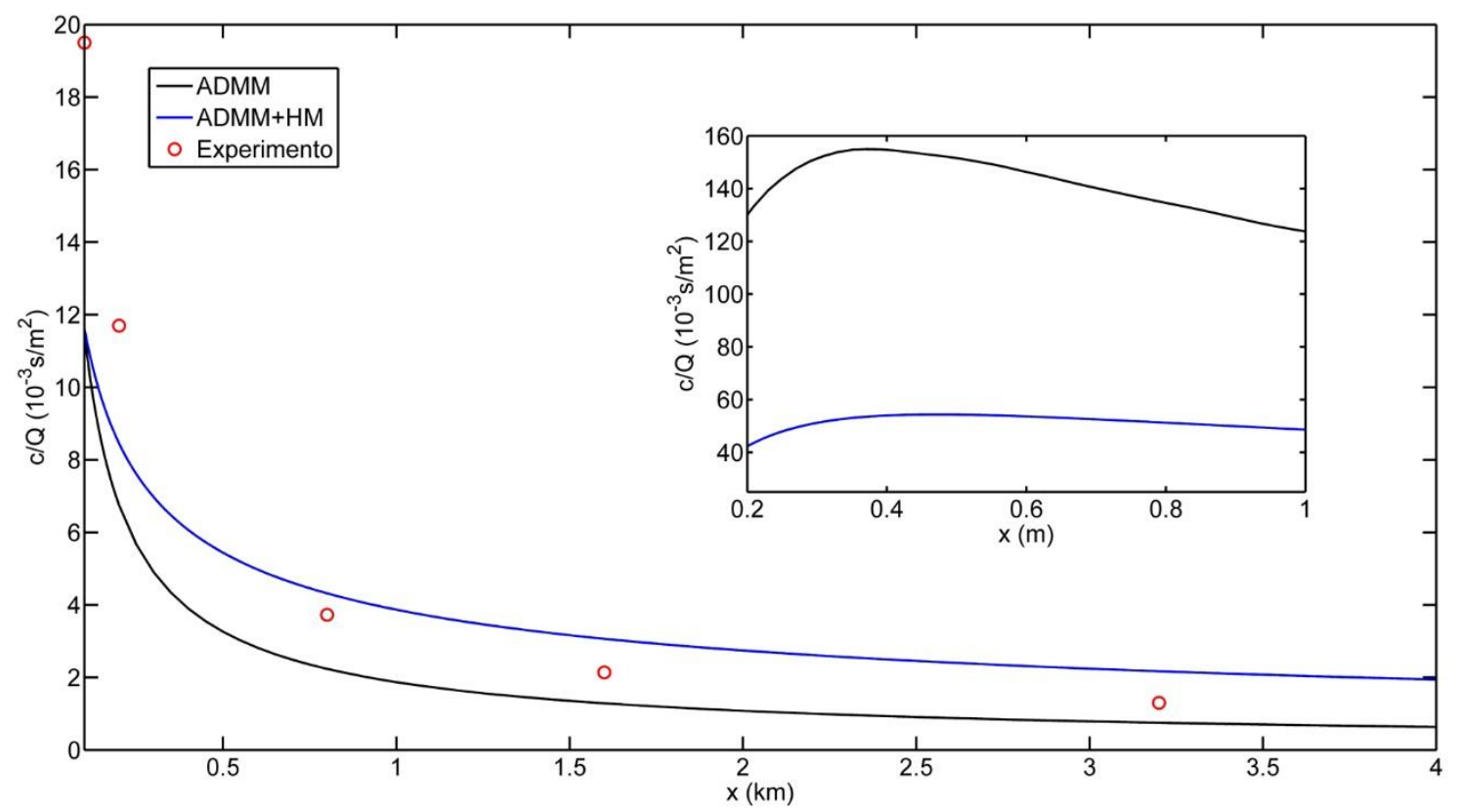

Figura 1: Comparação dos resultados da solução ADMM (16)-(17) e a aproximação por homogeneização matemática (26)-(27) das simulações computacionais junto com as observações do experimento de Hanford

Para avaliar a precisão dos resultados obtidos via a abordagem proposta em comparação com os resultados obtidos via o ADMM e as observações do experimento de Hanford, o desempenho de ambos os métodos é medido em termos dos índices estatísticos propostos em [19], a saber, o erro quadrático médio normalizado (NMSE), o coeficiente de correlação (COR), o fator de dois (FA2), o viés fracionário (FB), e o desvio padrão fracionário (FS). Estes índices estatísticos são calculados como segue, em que a barra indica a média aritmética e $\sigma$ denota o desvio padrão:

$$
\begin{aligned}
& \text { NMSE }=\frac{\overline{\left(c_{p}-c_{o}\right)^{2}}}{\overline{c_{p} c_{o}}}, \\
& \text { COR }=\frac{\overline{\left(c_{o}-\bar{c}_{o}\right)\left(c_{p}-\bar{c}_{p}\right)}}{\overline{\sigma_{o} \sigma_{p}}}, \\
& F A 2=(\text { percentual de }) \frac{c_{p}}{c_{o}} \in[0.5,2], \\
& F B=\frac{\bar{c}_{o}-\bar{c}_{p}}{0.5\left(\bar{c}_{o}+\bar{c}_{p}\right)}, \\
& F S=\frac{\sigma_{o}-\sigma_{p}}{0.5\left(\sigma_{o}+\sigma_{p}\right)} .
\end{aligned}
$$


A Tabela 1 apresenta os valores dos índices estatísticos (28)-(32) dos resultados das simulações computacionais apresentados na Fig. 1. Como esperado, observa-se que a precisão da abordagem proposta é inferior à do ADMM, mas aceitável, sobre tudo quando se considera o ganho em tempo na disponibilização dos resultados, o qual é muito útil para avaliações iniciais em situações de emergência.

Tabela 1: Índices estatísticos em comparação com o experimento de Hanford (valores ideais)

\begin{tabular}{cccccc}
\hline Método & NMSE (0) & COR (1) & FA2 (1) & FB (0) & FS (0) \\
\hline ADMM+MH & 0.06 & 0.939 & 0.889 & -0.134 & 0.160 \\
ADMM & 0.12 & 0.866 & 0.722 & -0.176 & 0.035 \\
\hline
\end{tabular}

Ainda, tal perda de precisão pode ser superada considerando outros aspectos da homogeneização matemática. Com efeito, aqui se considerou apenas a aproximação de ordem zero, isto é, a solução do problema homogeneizado. Seguindo a metodologia da homogeneização assintótica, é possível construir soluções assintóticas de qualquer ordem, o qual permite ter boas aproximações da solução exata do problema original com a precisão desejada.

\section{CONCLUSÃO}

Neste trabalho, para acelerar a disponibilidade de resultados com a menor perda de exatidão possível, apresentou-se a combinação do ADMM com homogeneização matemática. Segundo nosso melhor conhecimento, a aplicação da homogeneização matemática à modelagem da dispersão de poluentes na atmosfera parece ser uma novidade na área. Para avaliar o desempenho da abordagem proposta combinando o ADMM e homogeneização matemática, foram realizadas simulações computacionais cujos resultados foram comparados com os resultados da aplicação direta do ADMM e as observações do experimento de Hanford. Nas diversas simulações realizadas observou-se que a aplicação direta do ADMM, o qual apresenta o menor custo computacional quando comparado com outros métodos baseados em transformadas integrais, consumiu $\sim 15-20$ vezes mais tempo computacional que a combinação do ADMM com a homogeneização matemática. Este fato é relevante tanto quando se lida com uma grande quantidade de dados, quanto em avaliações iniciais em situações de emergência, $o$ qual compensa a pequena perda de precisão observada.

\section{AGRADECIMENTOS}

Os autores agradecem o projeto CAPES No. 88881.030424/2013-01 pelo suporte financeiro.

\section{REFERÊNCIAS BIBLIOGRÁFICAS}

1. Costa CP, Vilhena MT, Moreira DM, Tirabassi T. Semi-analytical solution of the steady threedimensional advection-diffusion equation in the planetary boundary layer. Atmos Environ. 2006;40:5659-5669, doi: 10.1016/j.atmosenv.2006.04.054.

2. Moreira DM, Vilhena MT, Tirabassi T, Buske D, Costa CP. Comparison between analytical models to simulate pollutant dispersion in the atmosphere. Int J Environ Waste Manage. 2010;6:327-344, doi: 10.1504/IJEWM.2010.035066.

3. Moreira DM, Vilhena MT, Buske D, Tirabassi T. The state-of-art of the GILTT method to simulate pollutant dispersion in the atmosphere. Atmos Res. 2009;92:1-17, doi: 10.1016/j.atmosres.2008.07.004.

4. Bensoussan A, Lions JL, Papanicalou G. Asymptotic analysis for periodic structures. Amsterdam: North-Holland; 1978. 699 p.

5. Pobedrya BE. Mechanics of composite materials. Moscow: Moscow State University Press; 1984. 336 p. (em russo)

6. Bakhvalov N, Panasenko G. Homogenisation: averaging processes in periodic media. Dordrecht: Kluwer Academic Publishers; 1989. 366 p. 
7. Arya SP. Modeling and parameterization of near source diffusion in weak winds. J App Meteor. 1995;34:1112-1122, doi: 10.1175/1520-0450(1995)034<1112:MAPONS > 2.0.CO;2.

8. Hanna SR. Atmospheric Turbulence and Air Pollution Modelling. Holland (D. Reidel Publishing Company, Dordrecht); 1982. Chapter 7, Applications in air pollution modeling; p. 275-310, doi: 10.1007/978-94-010-9112-1_7.

9. Panofsky HA, Dutton JA. Atmospheric turbulence: models and methods for engineering applications. New York : John Wiley \& Sons; 1984. 397 p.

10. Irwin JS. A theoretical variation of the wind profile power-law exponent as a function of surface roughness and stability. Atmos Environ. 1979;13:191-194, doi: 10.1016/0004-6981(79)90260-9.

11. Essa, K.S.M., Etman, S.M., Embaby, M., 2007. New analytical solution of the dispersion equation. Atmospheric Research 84, 337-344, doi: 10.1016/j.atmosres.2006.09.005

12. Abate J, Valkó PP. Multi-precision Laplace transform inversion. Int J Numer Methods Eng. 2004;60:979-993, doi:10.1002/nme.995.

13. Moreira DM, Moraes AC, Goulart AG, Albuquerque TTA. A contribution to solve the atmospheric diffusion equation with eddy diffusivity depending on source distance. Atmos Environ. 2014;83:254259, doi: 10.1016/j.atmosenv.2013.10.045.

14. Pao CV. Nonlinear parabolic and elliptic equations. New York : Plenum; 1992. 777 p.

15. Tartar L. The general theory of homogenization: a personalized introduction. Springer-Verlag Berlin Heidelberg; 2009. 470 p.

16. Gorbachev VI, Pobedrya BE. The effective characteristics of inhomogeneous media. J Appl Math Mech. 1997;61:145-151, doi: 10.1016/S0021-8928(97)00017-8.

17. Cioranecu D, Donato P. An introduction to homogenization. New York: Oxford University Press; 1999. $262 \mathrm{p}$.

18. Doran JC, Abbey OB, Buck JW, Glover DW, Horst TW, Lee RN, Lloyd FD. Field validation of exposure assessment models. United States: Environmental Protection Agency, Environmental Sciences Research Laboratory; 1984. 177 p.

19. Hanna SR. Confidence limits for air quality model evaluations, as estimated by bootstrap and jackknife resampling methods. Atmos Environ. 1989;23:1385-1398, doi: 10.1016/00046981(89)90161-3. 